\title{
Plasmonic Superlensing in Doped GaAs
}

\author{
Markus Fehrenbacher, ${ }^{*}{ }^{\dagger, \ddagger}$ Stephan Winnerl, ${ }^{* \dagger}$ Harald Schneider, ${ }^{\dagger}$ Jonathan Döring, ${ }^{\ddagger}$ Susanne C. Kehr, \\ Lukas M. Eng, Yongheng Huo, ${ }^{\S}$ Oliver G. Schmidt, ${ }^{\S}$ Kan Yao, ${ }^{\ddagger}$ Yongmin Liu, ${ }^{\|}$and Manfred Helm ${ }^{\dagger, \ddagger}$ \\ ${ }^{\dagger}$ Helmholtz-Zentrum Dresden-Rossendorf, Institute of Ion Beam Physics and Materials Research, Bautzner Landstraße 400, 01328 \\ Dresden, Germany \\ ${ }^{\ddagger}$ Institut für Angewandte Physik, TU Dresden, 01062 Dresden, Germany \\ ${ }^{\S}$ Institute for Integrative Nanosciences, IFW Dresden, Helmholtzstrasse 20, 01069 Dresden, Germany \\ ${ }^{\|}$Department of Electrical and Computer Engineering, Northeastern University, Boston, Massachusetts 02115, United States
}

ABSTRACT: We demonstrate a semiconductor based broadband near-field superlens in the mid-infrared regime. Here, the Drude response of a highly doped $\mathrm{n}$-GaAs layer induces a resonant enhancement of evanescent waves accompanied by a significantly improved spatial resolution at radiation wavelengths around $\lambda=20$ $\mu \mathrm{m}$, adjustable by changing the doping concentration. In our experiments, gold stripes below the GaAs superlens are imaged with a $\lambda / 6$ subwavelength resolution by an apertureless near-field optical microscope utilizing infrared radiation from a free-electron laser. The resonant behavior of the observed superlensing effect is in excellent agreement with simulations based on the Drude-Lorentz model. Our results demonstrate a rather simple superlens implementation for infrared nanospectroscopy.

KEYWORDS: Superlens, diffraction limit, surface plasmons, near-field microscopy, semiconductor

$\mathrm{I}_{\mathrm{stan}}^{\mathrm{n}}$ classical optical microscopy, spatial resolution is constrained by the wavelength of the applied radiation, limited by diffraction. In 2000, Pendry proposed that a negative refractive-index material ${ }^{1}$ could act as a perfect lens, ${ }^{2}$ which not only focuses propagating waves but in addition reconstructs information contained in the evanescent fields of an object, thereby creating an image with a resolution beyond the diffraction limit. Pendry also showed that in the near-field regime, negative permittivity alone is sufficient to realize a superlens to recover details on a subwavelength scale, which initiated a number of related experiments. While thin planar silver sheets have been demonstrated to be promising candidates for superlens-based UV nanophotolithography, ${ }^{3-5}$ near-field investigations of $\mathrm{SiC}^{6}{ }^{6}$ perovskites $^{7,8}$ and graphene ${ }^{9}$ reveal imaging capabilities beyond the diffraction limit at infrared wavelengths. Depending on the material, the spectral position and bandwidth of superlensing is determined by its plasma frequency $^{3-5,9}$ and phonon resonances. ${ }^{6-8}$ Consequently, accessible wavelengths are restricted by the limited availability of suitable materials. Various approaches have been proposed to overcome these restrictions. On the one hand, multilayered systems with different phonon resonances ${ }^{10}$ and the concept of an "unmatched superlens"11 promise to broaden the operation wavelength range of a superlens. On the other hand, the superlensing wavelength can be tailored by manipulating the electronic properties and, thus, the permittivity of a material. Correspondingly it has been suggested to use doped graphene ${ }^{12}$ or metal-dielectric composites $^{13}$ as frequency-adjustable subdiffractive imaging systems, continuously covering the visible and infrared range by controlling the respective plasma frequencies. Especially relevant to this work, semiconductors have been suggested to be exploited as plasmonic devices ${ }^{14-17}$ where the operational spectral range can be adapted by changing the doping level. However, a superlens consisting of doped semiconductor has not been demonstrated yet.

Our approach to realize a spectrally adjustable plasmonic superlens in the mid- and far-infrared is to use Si-doped GaAs, taking advantage of precisely controllable charge-carrier concentration by standard semiconductor fabrication techniques. Here, the electron density determines the plasma frequency of a conductive layer which in turn determines the superlensing wavelength. In this article, we evidence the performance of such a device by imaging gold stripes below the superlens with a scattering-type scanning near-field optical microscope $(\mathrm{s}-\mathrm{SNOM})^{18}$ combined with a free-electron laser (FEL). ${ }^{7,8,19-21}$ The observed enhancement of both the nearfield signal and the spatial resolution is consistent with theoretical considerations based on the Drude-Lorentz model, taking into account free electrons and optical phonons. $^{22}$

Superlensing for evanescent electric fields is related to the excitation of strongly localized surface polariton modes ${ }^{23}$ at the interface between two media, $\mathrm{A}$ and $\mathrm{B}$, with permittivities of

Received: October 17, 2014

Revised: January 8, 2015

Published: January 13, 2015 
same value and opposite sign, ${ }^{24}$ that is, $\operatorname{Re}\left(\varepsilon_{\mathrm{A}}\right)=-\operatorname{Re}\left(\varepsilon_{\mathrm{B}}\right)$. In our case, subwavelength resolved images arise due to coupled surface plasmon polariton modes confined at the interfaces between a doped semiconductor layer (B) and its neighboring intrinsic layers (A), enhancing the transfer of evanescent waves through the multilayer slab. This superlens structure (cf. Figure 1) consists of a highly n-doped ( $\mathrm{Si}$ ) GaAs film of thickness $d$

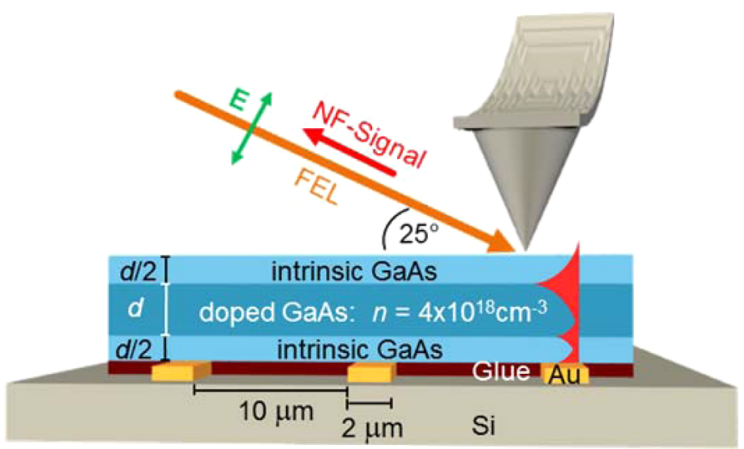

Figure 1. s-SNOM investigation of a GaAs superlens: an AFM tip is illuminated by p-polarized FEL radiation, the scattered light yielding information about the local near-field (NF) of the sample. Gold stripes (width $2 \mu \mathrm{m}$, spacing $10 \mu \mathrm{m}$ ) are imaged through the three-layered system. For the investigated samples, the layer thickness $d$ equals 200 and $400 \mathrm{~nm}$, respectively. The red feature in the sample indicates the distribution of the evanescent field along the surface normal in a similar manner as in ref 6 .

sandwiched between two undoped layers of thickness $d / 2$. Two superlens samples were prepared with a total thickness $2 d$ of 400 and $800 \mathrm{~nm}$, respectively. Additionally, a reference sample consisting of an intrinsic $400 \mathrm{~nm}$ thick GaAs layer was investigated. The object to be imaged is a periodic arrangement of gold stripes of $2 \mu \mathrm{m}$ width at a spacing of $10 \mu \mathrm{m}$. All samples were prepared in the following way: At first, a $100 \mathrm{~nm}$ thick $\mathrm{Al}_{0.9} \mathrm{Ga}_{0.1}$ As etch-stop layer was grown by molecular beam epitaxy (MBE) on a GaAs substrate, followed by the three GaAs layers $(A-B-A)$ of the superlens. On top of the last intrinsic layer, the gold stripes were patterned by optical lithography, gold deposition, and lift-off processing. Subsequently, the entire system was glued top-down by instant adhesive onto high-resistive silicon for the ease of handling. Then the GaAs substrate was etched away by citric acid down to the $\mathrm{Al}_{0.9} \mathrm{Ga}_{0.1}$ As etch stop, which was finally removed by concentrated $\mathrm{HCl}$.

In order to deduce the dielectric function of the superlens layers, Fourier transform infrared spectroscopy (FTIR) was performed in reflection geometry on the MBE grown samples prior to the lithography step (i.e., on the layer sequence GaAs/ $\mathrm{n}$-GaAs/GaAs/AlGaAs on GaAs substrate). Within a large part of the infrared electromagnetic spectrum, the relative permittivity $\varepsilon$ can be described well by the Drude-Lorentz model $^{22}$

$$
\varepsilon(\omega)=\varepsilon_{\text {optic }}-\frac{\left(\varepsilon_{\text {static }}-\varepsilon_{\text {optic }}\right) \omega_{\mathrm{TO}}^{2}}{\omega^{2}-\omega_{\mathrm{TO}}^{2}+i \omega \gamma_{\mathrm{ph}}}-\frac{\varepsilon_{\mathrm{optic}} \omega_{\mathrm{p}}^{2}}{\omega^{2}+i \omega \gamma_{\mathrm{el}}}
$$

where $\varepsilon_{\text {static }}$ and $\varepsilon_{\text {optic }}$ are the static and high-frequency dielectric constants, respectively. While values for the transverse optical phonon frequency $\omega_{\mathrm{TO}}$ and the phonon damping $\gamma_{\mathrm{ph}}$ are well known from the literature, ${ }^{22}$ the plasma frequency $\omega_{\mathrm{p}}$ (and thus the charge carrier density $n$ ) and the electron damping $\gamma_{\mathrm{el}}$ of the doped GaAs layer were determined by fitting a Drude-Lorentz based multilayer reflectivity simulation to the FTIR data. This results in $n=4 \times 10^{18} \mathrm{~cm}^{-3}$ and $\gamma_{\mathrm{el}}=100 \mathrm{~cm}^{-1}$. In addition, $n$ was confirmed by a Hall measurement in van der Pauw geometry. Using these values the infrared permittivities $\varepsilon_{\mathrm{n} \text {-GaAs }}$ and $\varepsilon_{\mathrm{GaAs}}$ of both the conducting and intrinsic GaAs layers were calculated (cf. Figure 2), which govern the spectral response of

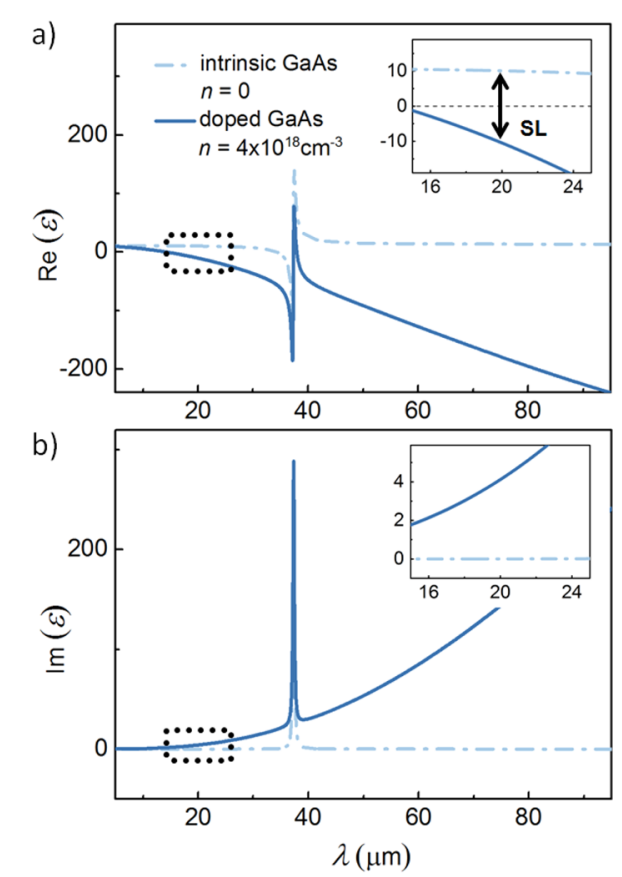

Figure 2. (a) Real part of the dielectric function $\operatorname{Re}(\varepsilon)$ of intrinsic and doped GaAs, as determined by FTIR measurements, fulfilling the superlensing condition $\operatorname{Re}\left(\varepsilon_{\mathrm{n}-\mathrm{GaAs}}\right)=-\operatorname{Re}\left(\varepsilon_{\mathrm{GaAs}}\right)$ at $\lambda \sim 20 \mu \mathrm{m}$ (see inset). (b) Imaginary part of the dielectric function $\operatorname{Im}(\varepsilon)$. At the superlensing wavelength $\operatorname{Im}\left(\varepsilon_{\mathrm{GaAs}}\right) \sim 0$ and $\operatorname{Im}\left(\varepsilon_{\mathrm{n}-\mathrm{GaAs}}\right) \sim 4$. The value for phonon damping, $\gamma_{\mathrm{ph}}=2 \mathrm{~cm}^{-1}$, is taken from ref 22 .

the superlens. Although the real part $\operatorname{Re}(\varepsilon)$ of intrinsic GaAs, apart from the Reststrahlen band around $\lambda=37 \mu \mathrm{m}$, is fairly constant in the infrared, the introduction of electrons results in increasingly negative values for longer wavelengths due to freecarrier absorption (cf. Figure 2a). Superlensing is expected for $\operatorname{Re}\left(\varepsilon_{\mathrm{n} \text {-GaAs }}\right)=-\operatorname{Re}\left(\varepsilon_{\mathrm{GaAs}}\right)$, which is the case for our sample at $\lambda$ $\sim 20 \mu \mathrm{m}$ (cf. Figure 2a, inset). At this wavelength, the imaginary part $\operatorname{Im}(\varepsilon)$ (cf. Figure $2 b$ ) of both intrinsic and doped GaAs is comparibly small corresponding to small dissipation, which is a critical parameter for the performance of a superlens. Please note that the precisely controllable doping level $n$ of GaAs offers the potential to adjust the superlensing wavelength, which increases with decreasing free electron concentration.

The near-field signal of the gold stripes is recorded by sSNOM (obtained via noninterferometric detection of the backscattered light), which allows imaging with a resolution far below the diffraction limit. ${ }^{18}$ Here, an oscillating atomic force microscope (AFM) tip acts as a scatterer for incoming radiation, where the scattering efficiency is modified by tipsample interaction. Acting as a small antenna, the tip converts the local near-field of the sample into an accessible far-field signal. Scanning the sample in tapping mode, the oscillation of the tip allows us to separate the near-field signal from the farfield background by lock-in demodulation of the measured 
Optical micrograph

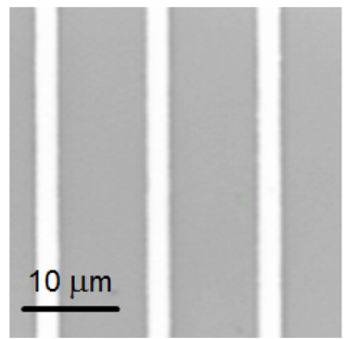

b)

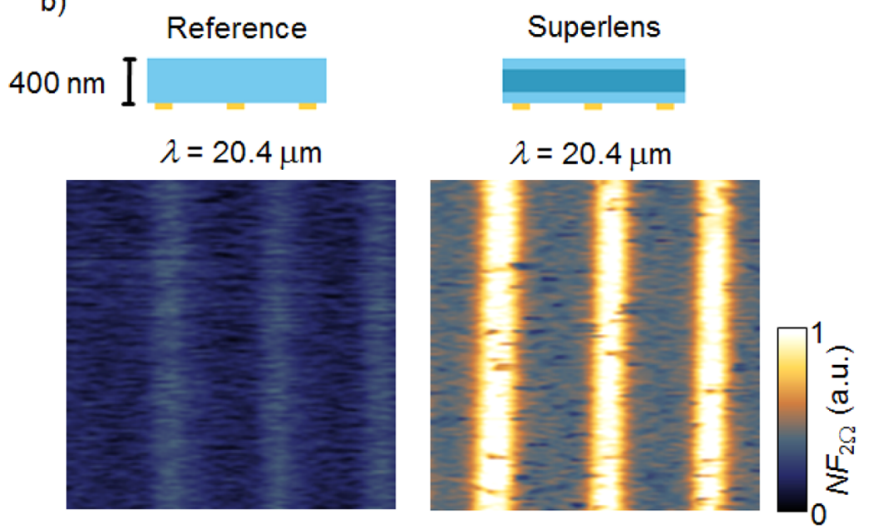

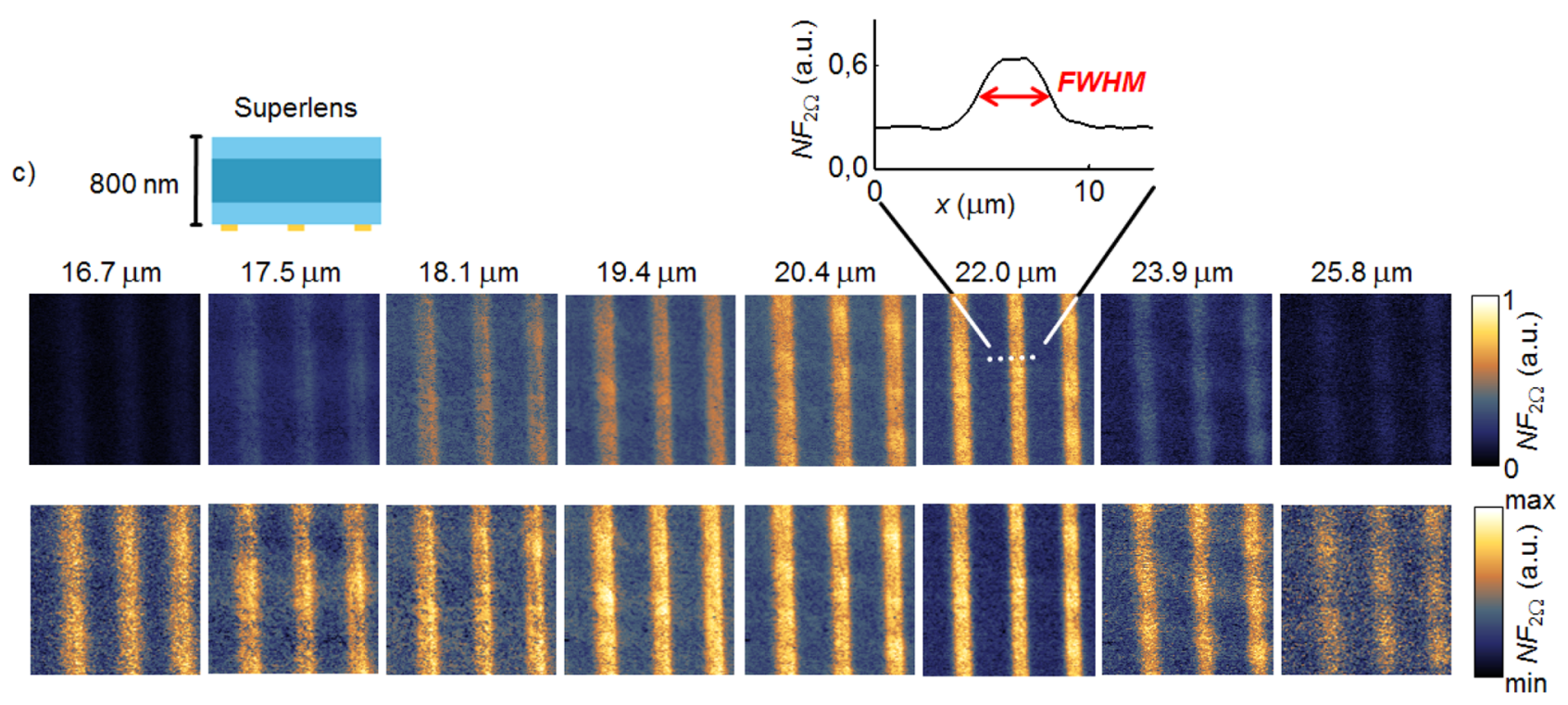

Figure 3. (a) Image of the stripes by optical microscopy for comparison, taken before the pattern was covered by the superlens. (b) Near-field images $\left(\mathrm{NF}_{2 \Omega}\right)$ of gold stripes below $400 \mathrm{~nm}$ intrinsic GaAs as a reference (left) and below a superlens with the same total thickness, including 200 $\mathrm{nm}$ doped GaAs (right). (c) Upper row: near-field images of gold stripes below an $800 \mathrm{~nm}$ thick superlens (intrinsic GaAs: $2 \times 200 \mathrm{~nm}$, doped GaAs: $400 \mathrm{~nm}$ ), recorded at radiation wavelengths from $\lambda=16.7 \mu \mathrm{m}$ to $\lambda=25.8 \mu \mathrm{m}$. For $\lambda=22.0 \mu \mathrm{m}$ a line profile with a full width at half-maximum (fwhm) of $3.4 \mu \mathrm{m}$ is shown. Bottom row: same measurements but with a full-range color scale applied to each image.

signal at higher harmonics of the cantilever's oscillation frequency $\Omega .{ }^{25}$ The near-field images in this study were obtained by $2 \Omega$ demodulation, denoted as $\mathrm{NF}_{2 \Omega}$. As a radiation source we employ the widely tunable FEL (radiation wavelength $4-250 \mu \mathrm{m}$ ) at the Helmholtz-Zentrum Dresden-Rossendorf (HZDR). The scattered radiation is detected in backward direction by a mercury-cadmium-telluride (MCT) detector.

In the experiment, we first compare the near-field of the gold-stripe pattern underneath the $400 \mathrm{~nm}$ thick superlens and underneath the $400 \mathrm{~nm}$ thick intrinsic GaAs reference structure. The measurement was performed at a wavelength of $\lambda=20.4$ $\mu \mathrm{m}$, which is close to the wavelength where superlensing is expected. As shown in Figure 3b, the stripes are barely visible in the image obtained from the reference sample. In this case, the $400 \mathrm{~nm}$ thick intrinsic GaAs layer reduces both the near-field signal and the spatial resolution of the near-field image ${ }^{26}$ due to the increased distance between SNOM tip and object. For the doped sample, the signal is highly increased, which provides clear evidence for superlensing. If one did not consider the superlensing effect, one would expect even weaker near-field signals for the sample with the doped layer as compared to the intrinsic reference sample because the doping results in freecarrier absorption that reduces the transmitted field. Nevertheless, absorption is one of the key factors that hinders the formation of a "perfect image". This applies to every superlens, as the requirement of negative permittivity is always accompanied by dissipation. In our case, the $2 \mu \mathrm{m}$ gold stripes are imaged with an apparent width of about $3 \mu \mathrm{m}$, clearly beating the diffraction limit. We emphasize that both for the reference sample and for the sample with superlens, the spatial resolution is not as good as for imaging the objects directly. However, for buried structures, introducing a doped layer into the capping enables us to image with drastically increased contrast while maintaining subwavelength resolution.

Note that the image obtained via the $400 \mathrm{~nm}$ thick superlens is not purely induced by the superlensing effect, as can be seen by the nonvanishing near-field in case of the reference structure. In order to suppress this background we increased the thickness of the superlens for further investigations.

The spectral dependence of the superlensing effect was investigated with the superlens sample of doubled thickness $(800 \mathrm{~nm})$, as shown in Figure 3c. The upper row depicts nearfield signals normalized to laser power, detector response and 
lock-in sensitivity, providing quantitative comparability. The near-field signal is strongest at $\lambda=22 \mu \mathrm{m}$, clearly decreasing to both sides of the spectrum. In addition, the image is sharpest in this case, while it becomes blurred at shorter and longer wavelengths (cf. Figure 3c, bottom row). A line plot across one stripe reveals a full width at half-maximum $(f w h m)$ of $3.4 \mu \mathrm{m}$ at resonance. Since the width of the stripe itself $(2 \mu \mathrm{m})$ strongly contributes to the $f w h m$, the actual resolution must be better than $3.4 \mu \mathrm{m}$, corresponding to a subwavelength resolution of at least $\lambda / 6$. Note that the image of the $400 \mathrm{~nm}$ thick superlens (cf. Figure $3 \mathrm{~b}$ ) exhibits a higher near-field signal as compared to the $800 \mathrm{~nm}$ thick superlens (cf. Figure 3c) for the same wavelength $\lambda=20.4 \mu \mathrm{m}$. This can be attributed to less absorption in the thinner layer.

The performance of a near-field superlens can be analyzed by calculating the transmittance $T$ through a sequence of homogeneous dielectric films ${ }^{27}$ at normal incidence. Depicted as a function of the tangential wavenumber $k_{\mathrm{t}}$ and radiation wavelength $\lambda$, this is often referred to as the transfer function. The transfer function of our GaAs superlens is shown in Figure $4 \mathrm{a}$, assuming a superlens suspended in air. Accordingly, the

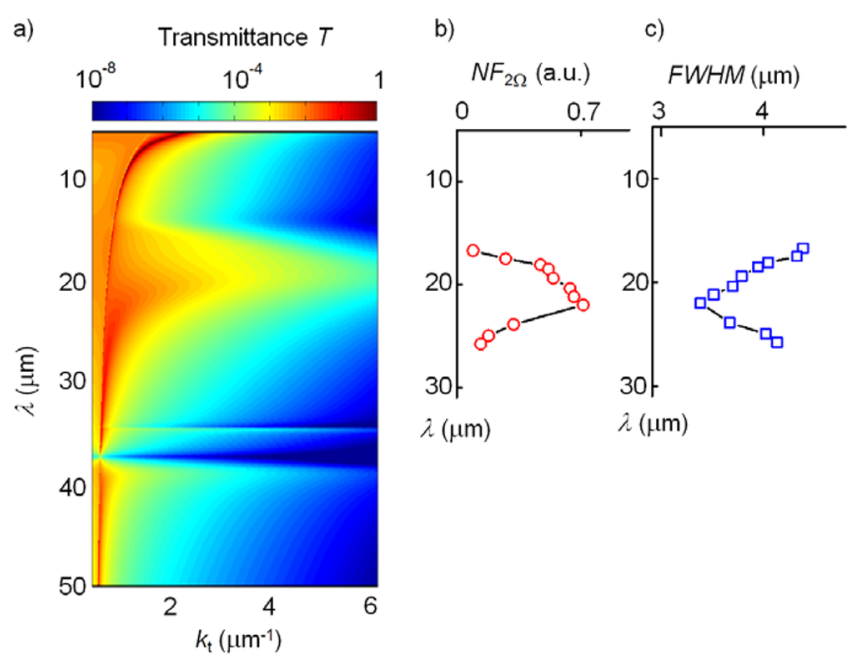

Figure 4. (a) Calculated transfer function $T$ of the GaAs superlens (800 nm total thickness) in the spectral range of interest, plotted in logarithmic scale for better visibility. (b) Experimental result: superlens enhanced near-field signal $N F_{2 \Omega}$ on gold stripes and (c) fwhm of a stripe's image as a function of radiation wavelength $\lambda$.

transmittance of large wavenumbers is resonantly enhanced around $\lambda=20 \mu \mathrm{m}$, illustrating the increase of imaging quality induced by the superlensing effect. This enhancement is consistent with the simple condition $\operatorname{Re}\left(\varepsilon_{\mathrm{n} \text {-GaAs }}\right)=-\operatorname{Re}\left(\varepsilon_{\mathrm{GaAs}}\right)$, which was described above. To relate the transfer function to our experimental findings, we also plot both the near-field signal $N F_{2 \Omega}$ at the position of a gold stripe (cf. Figure $4 \mathrm{~b}$ ) and the $f w h m$ of the stripe's image versus wavelength $\lambda$ (cf. Figure $4 \mathrm{c}$ ). Both the strongest signal and the smallest $f w h m$, according to the best spatial resolution, are observed at $\lambda=22 \mu \mathrm{m}$, as mentioned above. As can be seen, the resonant behavior is in very good agreement with theory.

The characterization of the subwavelength resolution of a superlens naturally requires a technique that itself provides even higher spatial resolution. In our case, this technique is $s$ $\mathrm{SNOM}^{18}{ }^{18}$ featuring a wavelength-independent resolution determined by the radius of the AFM tip $(<50 \mathrm{~nm})$. This is much smaller than the wavelength of the infrared radiation and also smaller than the observed resolution of the investigated superlens. Note, however, that the spatial resolution of SNOM significantly degrades with increasing depth for buried objects. ${ }^{26}$ In this context, the immediate application potential of our superlens lies in near-field imaging of structures for nanoelectronic applications which are necessarily covered by an intrinsic layer of a certain thickness, for example, to avoid depletion due to surface fields ${ }^{21,28}$ or imposed by the penetration depth of ion implantation. ${ }^{20,29}$ Here, the introduction of a doped layer turns the intrinsic layer into a superlens at a designed wavelength, enabling near-field studies of buried objects with enhanced sensitivity and better spatial resolution. For instance, nanoscopic imaging of buried quantum dots, ${ }^{21}$ doping profiles, ${ }^{20}$ and charge carriers in a field-effect transistor $^{30}$ can be significantly improved by this concept. Here, the semiconductor-based superlens can enhance the contrast of otherwise blurred images. In many cases, for example, for GaAs based III-V semiconductor compounds, even monolithic integration of the nanoobject and the superlens is possible. For such applications, a broad operation bandwidth may be desirable in order to access as many wavelengths as possible with one and the same structure. Notably, the relative spectral width $\Delta \lambda / \lambda_{\mathrm{SL}} \sim 25 \%$ ( $\lambda_{\mathrm{SL}}$ is the superlensing wavelength) of our plasmonic superlens is significantly larger as compared to previously investigated phonon based systems operating in the mid-infrared, where $\Delta \lambda / \lambda_{\mathrm{SL}}<10 \%$ was observed. ${ }^{6-8}$ On the one hand, the large bandwidth can be attributed to a slow change of $\operatorname{Re}\left(\varepsilon(\lambda) / \operatorname{Re}\left(\varepsilon\left(\lambda_{\mathrm{SL}}\right)\right.\right.$, determining how well the superlensing condition is fulfilled in the spectral proximity of the resonance. On the other hand, damping, which is comparatively large in our case, also broadens the peak in the transfer function. Performing the simulation with artificially reduced $\operatorname{Im}(\varepsilon)$ indicates that the latter aspect is the predominant one (note that large values of $\operatorname{Im}(\varepsilon)$ are accompanied by a decrease of imaging quality). For a deeper understanding, however, a more detailed analysis is required, which is beyond the scope of our study. As mentioned, the center wavelength can easily be tailored by changing the doping level of the intermediate layer. We chose a carrier concentration of $n=4 \times 10^{18} \mathrm{~cm}^{-3}$, which is very close to the upper limit for Si doping of GaAs. ${ }^{31}$ This results in a shortest possible superlensing wavelength of about $20 \mu \mathrm{m}$. The choice of high $n$ might be surprising at first sight because it leads to large freecarrier absorption, which appears to be undesirable as dissipation strongly impacts the imaging quality of a superlens. However, the critical parameter here is $\operatorname{Im}(\varepsilon)$ at the superlensing wavelength $\lambda_{\mathrm{SL}}$. An increase of $n$ has two consequences: On the one hand, it results in larger values of $\operatorname{Im}(\varepsilon)$ at a given wavelength $\lambda$. On the other hand, $\lambda_{\mathrm{SL}}$ decreases accompanied by a decrease of $\operatorname{Im}(\varepsilon)$ (cf. Figure $2 \mathrm{~b}$ ). Altogether calculations (not shown) reveal that high $n$ is preferable since the latter effect dominates. Nevertheless, $\operatorname{Im}(\varepsilon)$ does not increase drastically when going to longer $\lambda_{\mathrm{SL}}(\operatorname{Im}(\varepsilon) \sim 4,8$, and 12 at $\lambda_{\mathrm{SL}}=20,90$, and $140 \mu \mathrm{m}$, respectively). Therefore, we expect that superlensing in doped GaAs can be transferred well into the terahertz region by less doping. In order to explore shorter wavelengths, the concept of a frequency-adjustable plasmonic superlens can easily be realized with alternative systems providing higher plasma frequencies and, therefore, shorter superlensing wavelengths. In particular, it has recently been shown that the plasma wavelength of InAs can be controlled down to $\sim 5 \mu \mathrm{m}$, taking advantage of its small effective mass and the feasibility of extremely high doping. ${ }^{15,16}$ In addition, the 
spatial resolution of an InAs-based superlens should be better than for GaAs due to its high electron mobility. In the past few years, a lot of effort was put into the search for low-loss plasmonic materials at telecom or even optical wavelengths. ${ }^{14,17}$ In the near-infrared, transparent conducting oxides (TCOs) such as aluminum zinc oxide (AZO) or indium tin oxide (ITO) may be suitable as building blocks for spectrally adjustable superlenses, whereas in the optical regime, silver seems to be the best choice.

In summary, we have demonstrated a GaAs superlens, employing a sequence of intrinsic and doped layers to create subwavelength images in a broad mid-infrared spectral range. Best resolution, about one-sixth of the applied wavelength $\lambda$, of imaged gold stripes was observed at $\lambda=22 \mu \mathrm{m}$, which is in very good agreement with the calculated transfer function based on electronic properties of the doped layer extracted from FTIR reflection measurements. This plasmonic superlens with an adaptable operation wavelength is a versatile device to enhance signal and spatial resolution in near-field imaging of buried structures. Furthermore, it may find application in mid-infrared and terahertz devices that exploit plasmonic coupling of the radiation field to small structures.

\section{AUTHOR INFORMATION}

\section{Corresponding Authors}

*E-mail: m.fehrenbacher@hzdr.de. Phone: +49 3512603593. Fax: +49351 2603285.

*E-mail: s.winnerl@hzdr.de.

\section{Notes}

The authors declare no competing financial interest.

\section{ACKNOWLEDGMENTS}

We gratefully acknowledge the funding by the German Ministry of Education and Research (BMBF; Grant 05K10BRA and 05K10ODB). We also thank the whole ELBE team for their dedicated support. Special credit goes to Claudia Neisser and Jan Fiedler for preparation and characterization of the samples, respectively.

\section{REFERENCES}

(1) Veselago, V. G. Sov. Phys. Uspekhi 1968, 10, 509-514.

(2) Pendry, J. B. Phys. Rev. Lett. 2000, 85, 3966-3969.

(3) Melville, D. O. S.; Blaikie, R. J.; Wolf, C. R. Appl. Phys. Lett. 2004, 84, 4403-4405.

(4) Fang, N.; Lee, H.; Sun, C.; Zhang, X. Science 2005, 308, 534537.

(5) Liu, H.; Wang, B.; Ke, L.; Deng, J.; Chum, C. C.; Teo, S. L.; Shen, L.; Maier, S. A.; Teng, J. Nano Lett. 2012, 12, 1549-1554.

(6) Taubner, T.; Korobkin, D.; Urzhumov, Y.; Shvets, G.; Hillenbrand, R. Science 2006, 313, 1595.

(7) Kehr, S. C.; Yu, P.; Liu, Y. M.; Parzefall, M.; Khan, A. I.; Jacob, R.; Wenzel, M. T.; von Ribbeck, H.-G.; Helm, M.; Zhang, X.; Eng, L. M.; Ramesh, R. Opt. Mater. Express 2011, 1, 1051-1060.

(8) Kehr, S. C.; Liu, Y. M.; Martin, L. W.; Yu, P.; Gajek, M.; Yang, S.Y.; Yang, C.-H.; Wenzel, M. T.; Jacob, R.; von Ribbeck, H.-G.; Helm, M.; Zhang, X.; Eng, L. M.; Ramesh, R. Nat. Commun. 2011, 2, 249.

(9) Li, P.; Wang, T.; Böckmann, H.; Taubner, T. Nano Lett. 2014, 14, 4400-4405.

(10) Li, P.; Taubner, T. Opt. Express 2012, 20, A11787-A11795.

(11) Yang, X.; Liu, Y.; Ma, J.; Cui, J.; Xing, H.; Wang, W.; Wang, C.; Luo, X. Opt. Express 2008, 16, 19686-19694.

(12) Li, P.; Taubner, T. ASC Nano 2012, 6, 10107-10114.

(13) Cai, W.; Genov, D. A.; Shalaev, V. M. Phys. Rev. B 2005, 72, 193101.
(14) West, P. R.; Ishii, S.; Naik, G. V.; Emani, N. K.; Shalaev, V. M.; Boltasseva, A. Laser Photonics Rev. 2010, 4, 795-808.

(15) Law, S.; Adams, D. C.; Taylor, A. M.; Wasserman, D. Opt. Express 2012, 20, 12155-12165.

(16) Law, S.; Podolskiy, V.; Wasserman, D. Nanophotonics 2013, 2, 103-130.

(17) Naik, G. V.; Shalaev, V. M.; Boltasseva, A. Adv. Mater. 2013, 25, 3264-3294.

(18) Novotny, L.; Stranick, S. J. Annu. Rev. Phys. Chem. 2006, 57, 303-331.

(19) Kehr, S. C.; Cebula, M.; Mieth, O.; Härtling, T.; Seidel, J.; Grafström, S.; Eng, L. M.; Winnerl, S.; Stehr, D.; Helm, M. Phys. Rev. Lett. 2008, 100, 256403.

(20) Jacob, R.; Winnerl, S.; Schneider, H.; Helm, M.; Wenzel, M. T.; von Ribbeck, H.-G.; Eng, L. M.; Kehr, S. C. Opt. Express 2010, 18 26206-26213.

(21) Jacob, R.; Winnerl, S.; Fehrenbacher, M.; Bhattacharyya, J.; Schneider, H.; Wenzel, M. T.; von Ribbeck, H.-G.; Eng, L. M.; Atkinson, P.; Schmidt, O. G.; Helm, M. Nano Lett. 2012, 12, 43364340.

(22) Blakemore, J. S. J. Appl. Phys. 1982, 53, R123-R181.

(23) Dionne, J. A.; Sweatlock, L. A.; Atwater, H. A.; Polman, A. Phys. Rev. B 2005, 72, 075405 .

(24) Korobkin, D.; Urzhumov, Y.; Shvets, G. J. Opt. Soc. Am. B 2006, $23,468-478$

(25) Knoll, B.; Keilmann, F. Opt. Commun. 2000, 182, 321-328.

(26) Krutokhvostov, R.; Govyadinov, A. A.; Stiegler, J. M.; Huth, F.; Chuvilin, A.; Carney, P. S.; Hillenbrand, R. Opt. Express 2012, 20, 593-600.

(27) Born, M.; Wolf, E. Principles of Optics, $7^{\text {th }}$ ed.; Cambridge University Press: New York, 1999.

(28) Buchwald, W. R.; Cleary, J. W.; Hendrickson, J. Appl. Phys. Lett. 2012, 100, 051110

(29) Brice, D. K. Appl. Phys. Lett. 1970, 16, 103-106.

(30) Huber, A. J.; Keilmann, F.; Wittborn, J.; Aizpurua, J.; Hillenbrand, R. Nano Lett. 2008, 8, 3766-3770.

(31) Neave, J. H.; Dobson, P. J.; Harris, J. J.; Dawson, P.; Joyce, B. A. Appl. Phys. A: Mater. Sci. Process. 1983, 32, 195-200. 